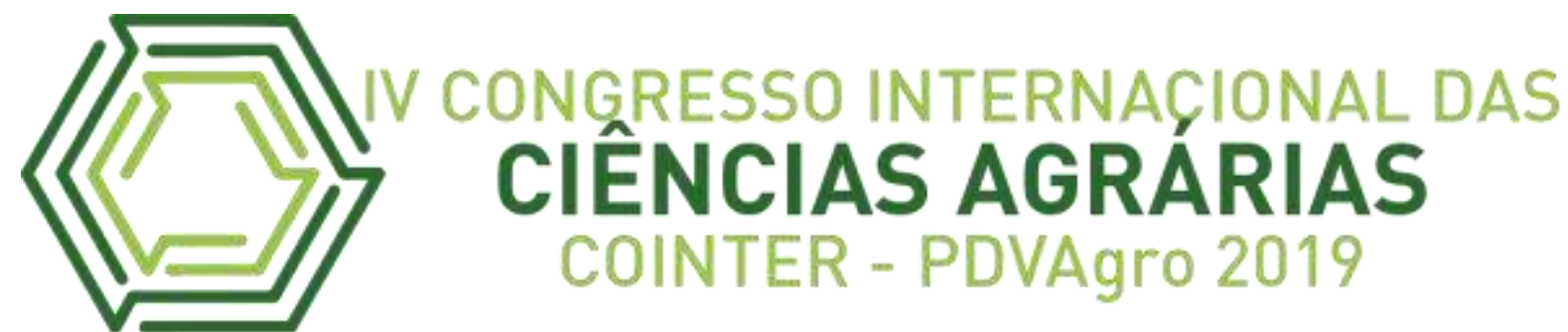

\title{
DEGRADAÇÃO DA VITAMINA C EM MAÇÃS DA VARIEDADE FUJI APÓS O BRANQUEAMENTO
}

\section{DEGRADACIÓN DE VITAMINA C EN MANZANAS DE FUJI VARIEDAD DESPUÉS DE BLANKING}

\section{VITAMIN C DEGRADATION IN FUJI VARIETY APPLES AFTER BLANKING PROCESS}

\author{
Apresentação: Comunicação Oral
}

Sonara de França Sousa ${ }^{1}$; Francilânia Batista da Silva² ${ }^{2}$ Krause Gonçalves Silveira Albuquerque $^{3}$; Gustavo Henrique Daniel Santos Silva ${ }^{4}$; Josivanda Palmeira Gomes ${ }^{5}$

DOI: https://doi.org/10.31692/2526-7701.IVCOINTERPDVAgro.2019.0159

\begin{abstract}
Resumo
A maçã é uma frutífera de clima temperado bastante apreciada tanto pelo consumo in natura como na sua forma processada. O branqueamento é um pré-processamento realizado em frutas e hortaliças destinadas às etapas de congelamento e desidratação, tendo como finalidade a inativação enzimática, entretanto, as variáveis tempo e temperatura empregados neste processo podem provocar perdas de nutrientes, entre eles, as vitaminas. O objetivo do presente trabalho é avaliar a perda da vitamina $\mathrm{C}$, visto que esta é bastante suscetível à degradação térmica $\mathrm{e}$ oxidativa, durante o branqueamento por imersão. A determinação da vitamina $C$ foi feita a cada 15 minutos de exposição das fatias de maçãs não branqueadas, branqueadas a $70^{\circ} \mathrm{C}$ por 5 minutos e branqueadas a $70^{\circ} \mathrm{C}$ por 10 minutos, através do método proposto pela AOAC que utiliza como reagente o 2,6-diclorofenol indofenol e uma solução de ácido oxálico a 4\%. De acordo com os resultados obtidos, a acidez e o pH das maçãs apresentam-se de acordo com a literatura, entretanto, o teor de vitamina $\mathrm{C}$ quantificado encontrou-se abaixo do esperado para esta frutífera. As maçãs apresentaram diferenças significativas entre os tratamentos sendo o maior teor observado na amostra sem branqueamento em relação as demais. De acordo com o tempo de branqueamento, as perdas quantificadas foram de 32,08, 32,35 e 19,54\% para as amostras não branqueadas, branqueadas a $70^{\circ} \mathrm{C}$ por 5 minutos e branqueadas a $70^{\circ} \mathrm{C}$ por 10 minutos, respectivamente, o que representa uma desvantagem, visto que esta perda é considerada um dos parâmetros para avaliar a eficiência do pré-processamento.
\end{abstract}

\footnotetext{
${ }^{1}$ Doutora em Engenharia de Processos, Universidade Federal de Campina Grande, sonara_franca@yahoo.com.br

2 Doutora em Engenharia de Processos, Universidade Federal de Campina Grande, francilania@live.com

${ }^{3}$ Graduando em Engenharia de Alimentos, Universidade Federal Rural de Pernambuco albuquerque.k.g@gmail.com

${ }^{4}$ Graduando em Engenharia de Alimentos, Universidade Federal Rural de Pernambuco, gd30440@gmail.com

${ }^{5}$ Professora do Programa de Pós-graduação em Engenharia de Processos, Universidade Federal de Campina Grande, josivanda@gmail.com
} 
Palavras-Chave: ácido ascórbico, pré-processamento, tempo, temperatura.

\begin{abstract}
Resumen
La manzana es un árbol frutal templado muy apreciado por su consumo fresco y su forma procesada. El blanqueo es un preprocesamiento realizado en frutas y verduras para las etapas de congelación y deshidratación, con el propósito de la inactivación enzimática, sin embargo, las variables de tiempo y temperatura utilizadas en este proceso pueden causar pérdidas de nutrientes, incluidas las vitaminas. El objetivo del presente trabajo es evaluar la pérdida de vitamina $\mathrm{C}$, ya que es muy susceptible a la degradación térmica y oxidativa durante el blanqueo. La determinación de la vitamina $\mathrm{C}$ se realizó cada 15 minutos de exposición de rodajas de manzana sin blanquear, blanqueadas a $70{ }^{\circ} \mathrm{C}$ durante 5 minutos y blanqueadas a $70^{\circ} \mathrm{C}$ durante 10 minutos, por el método propuesto por la AOAC que utiliza 2 2,6-diclorofenol indofenol y una solución de ácido oxálico al $4 \%$. Según los resultados obtenidos, la acidez y el pH de las manzanas se presentan de acuerdo con la literatura, sin embargo, el contenido cuantificado de vitamina $\mathrm{C}$ fue menor de lo esperado para esta fruta. Las manzanas mostraron diferencias significativas entre los tratamientos y el mayor contenido observado en la muestra sin blanquear en comparación con los demás. Según el tiempo de blanqueo, las pérdidas cuantificadas fueron $32.08,32.35$ y $19.54 \%$ para muestras sin blanquear, blanqueadas a $70^{\circ} \mathrm{C}$ durante 5 minutos y blanqueadas a $70{ }^{\circ} \mathrm{C}$ durante 10 minutos, respectivamente. , lo que representa una desventaja, ya que esta pérdida se considera uno de los parámetros para evaluar la eficiencia del preprocesamiento.
\end{abstract}

Palabras Clave: ácido ascórbico, preprocesamiento, tiempo, temperatura.

\begin{abstract}
Apple is a temperate fruit that is well appreciated for its fresh consumption and its processed form. Blanching is a pre-processing performed on fruits and vegetables for the freezing and dehydration stages, with the purpose of enzymatic inactivation, however, the time and temperature variables used in this process can cause nutrient losses, including vitamins. The objective of the present work is to evaluate the loss of vitamin $\mathrm{C}$, since it is very susceptible to thermal and oxidative degradation during bleaching. The determination of vitamin $\mathrm{C}$ was made every 15 minutes of exposure of unbleached apple slices, bleached at $70^{\circ} \mathrm{C}$ for 5 minutes and bleached at $70^{\circ} \mathrm{C}$ for 10 minutes, by the method proposed by the AOAC which uses 2 2,6dichlorophenol indophenol and a $4 \%$ oxalic acid solution. According to the results obtained, the acidity and $\mathrm{pH}$ of apples are presented according to the literature, however, the quantified vitamin $\mathrm{C}$ content was lower than expected for this fruit. Apples showed significant differences between treatments and the highest content observed in the unbleached sample compared to the others. According to the bleaching time, quantified losses were $32.08,32.35$ and $19.54 \%$ for unbleached samples, bleached at $70^{\circ} \mathrm{C}$ for 5 minutes and bleached at $70{ }^{\circ} \mathrm{C}$ for 10 minutes, respectively, which represents a disadvantage, since this loss is considered one of the parameters to evaluate the preprocessing efficiency.
\end{abstract}

Keywords: ascorbic acid, preprocessing, time, temperature. 


\section{Introdução}

A maçã (Malus domestica Borkh) é uma fruta de clima temperado de maior importância comercial para consumo in natura (MELLO \& JÚNIOR, 2004). Entretanto, o mercado interno deste produto ainda é pequeno, o que justifica a avaliação da aplicação de tecnologias que possam agregar valor comercial e incentivando o seu consumo.

O branqueamento é uma das técnicas mais utilizadas em frutas e hortaliças destinadas ao congelamento e desidratação. Neste método, os alimentos são aquecidos rapidamente, através de imersão em água quente ou jatos a vapor com temperatura predeterminada. De acordo com Vasconcelos \& Filho (2017), a temperatura usada no branqueamento varia de $70^{\circ}$ a $100^{\circ} \mathrm{C}$ e o tempo de exposição dos alimentos a este procedimento varia de alguns minutos, logo após realiza-se o resfriamento a fim de cessar o processo de cozimento desses vegetais.

Como vantagens do branqueamento Orso (2011) afirma que este método é capaz de prevenir o escurecimento enzimático que ocorre em frutas e hortaliças, além de conseguir eliminar resíduos agrotóxicos provenientes dos produtos agrícolas ou até mesmo do próprio metabolismo vegetal. Entretanto, Sucupira et al. (2012), relatam que o alimento está sujeito a perdas de nutrientes, tais como algumas vitaminas hidrossolúveis.

A vitamina $\mathrm{C}$ ou, simplesmente, ácido ascórbico (AA) está amplamente distribuída nos produtos de origem vegetal, e é considerada uma vitamina hidrossolúvel e termolábil (ZHANG \& HAMAUZU, 2004). Sabe-se que diversos fatores afetam a estabilidade do ácido ascórbico durante o processamento e armazenamento, incluindo o $\mathrm{pH}$ do meio, a presença de oxigênio e de íons metálicos, e a temperatura (TARRAGO-TRANI et al., 2012; SPINOLA et al., 2013) e que trabalhos relacionados à degradação da vitamina $\mathrm{C}$ durante o processamento de alimentos vegetais já foram registrados em várias investigações (FERNÁNDEZ et al., 2011; GEORGÉ et al., 2011) no entanto pouco se sabe sobre as etapas que antecedem o processamento. Este trabalho teve como objetivo, avaliar a perda de vitamina $\mathrm{C}$ em maçãs variedade fuji submetidas ao branqueamento por imersão em água e expostas as condições ambientais.

\section{Fundamentação Teórica}

As frutas e vegetais, em sua maioria, são alimentos perecíveis e de produção sazonal, tornando-se inviável sua disponibilidade durante todo o ano, além de possuírem um tempo de 
vida útil curto. Devido a estes fatores, é comum sofrerem processamento industrial para então serem comercializadas sob a forma de sucos, polpas, doces, etc. No entanto, grandes proporções de vitamina $\mathrm{C}$ são perdidas no momento de pré-processamento, como é o caso da etapa de branqueamento (LAVARDA, 2011).

A vitamina $\mathrm{C}$ possui diversas funções, entre as principais pode-se citar a de antioxidante, devido a sua capacidade de ceder elétrons, é necessária para a síntese e manutenção do colágeno e também está envolvida na síntese de importantes neurotransmissores como a norepinefrina obtida a partir da dopamina e a serotonina, obtida pela conversão de triptofano em 5hidroxitriptofano (REBOUCHE, 1991; NAGAOKA et al., 2007; KALIORA et al, 1996). Essa vitamina atua na diminuição do risco de doenças cardiovasculares, no tratamento de hipertensão e na redução da incidência de cataratas. Estudos recentes também indicam que a vitamina $C$ pode atuar na prevenção do câncer, pois acredita-se que exista uma relação inversa entre frequência de tumores e consumo de alimentos ricos nesta vitamina. A vitamina $\mathrm{C}$ também é recomendada como suplemento alimentar, pois auxilia a absorção de minerais como ferro e zinco.

Na tecnologia de alimentos, busca-se a maximização da retenção dos nutrientes durante o processamento e o armazenamento dos produtos. O ácido ascórbico é um importante indicador, pois sendo a vitamina mais termolábil, sua presença no alimento indica que, provavelmente, os demais nutrientes também estão sendo preservados (ALVES et al., 2010).

Por isso, é considerado como indicativo de qualidade dos nutrientes durante o processamento e o armazenamento de alimentos. A estabilidade da vitamina $\mathrm{C}$ é dependente tanto do tipo de processamento quanto da temperatura de armazenagem, apresentando inclusive cinéticas de degradação diferenciadas, a estabilidade aumenta com a diminuição da temperatura do meio, embora existam casos de perda durante o congelamento, ou armazenamento de alimentos a temperatura de refrigeração (TAVARES et al., 2000; YAMASHITA, et al., 2003).

Trabalhos sobre a degradação da vitamina $\mathrm{C}$ durante o processamento de frutas são amplamente encontrados na literatura, a saber, Maia (2007) estudou a degradação de vitamina $\mathrm{C}$ na polpa de acerola e constatou que mesmo ocorrendo perdas significativas de vitamina $\mathrm{C}$ na polpa após seu processamento, o teor de vitamina $\mathrm{C}$ ainda era superior ao consumo diário para humanos. 
Teixeira e Monteiro (2004) argumentam que a exposição dos sucos de fruta ao oxigênio e à luz pode influir na sua qualidade, podendo reduzir o conteúdo de vitamina $\mathrm{C}$ e modificar sensorialmente o produto. Danieli et al. (2009) avaliaram a estabilidade da vitamina C em sucos de laranja industrializados e naturais e verificaram que os sucos in natura foram estáveis por até $14 \mathrm{~h}$ de armazenamento refrigerado, enquanto os industrializados não apresentaram perda significativa de ácido ascórbico por até 72h.

Umme et al. (1997) avaliaram as características físico-químicas do purê de graviola e verificaram que após a pasteurização no intervalo de 50 a $90{ }^{\circ} \mathrm{C}$ ocorreram perdas no teor de vitamina C da ordem de 51 a $87 \%$, respectivamente. Spinola et al. (2013) avaliaram o teor de vitamina $\mathrm{C}$ em extratos de maracujá, por 5 horas, em temperatura de $23^{\circ} \mathrm{C}$. Segundo esses autores, não houve queda mensurável da concentração da vitamina após $2 \mathrm{~h}$ de armazenamento, porém foram detectadas após 5 h de armazenamento no extrato de maracujá perdas de 6,3\%.

Diante do exposto, verifica-se que pesquisas relacionadas à degradação deste componente durante a etapa de branqueamento de frutas são inexistentes, surgindo a necessidade de levantamento de dados científicos para se obter uma melhor relação entre os processos que envolvem a elevação de temperatura com a perda da vitamina $C$.

\section{Metodologia}

O presente trabalho foi realizado no Laboratório de Armazenamento e Processamento de Produtos Agrícolas (LAPA), na Unidade Acadêmica de Engenharia Agrícola da Universidade Federal de Campina Grande-PB (UFCG). As maçãs da variedade fuji foram adquiridas no comercio local da cidade de Campina Grande (PB). A seleção das frutas foi feita usando como principal critério o grau de maturação (coloração da casca) onde as mesmas apresentaram-se adequadas para o consumo e processamento, sendo posteriormente lavadas em água corrente, sanitizadas em hipoclorito de sódio 200 ppm por dez minutos e secas com o auxílio de papel toalha.

O branqueamento foi realizado através da imersão das maçãs em água destilada a uma temperatura de $70^{\circ} \mathrm{C}$ por um período de tempo de 5 e 10 minutos, utilizando um banho-maria. Transcorrido o período de tempo estabelecido, as maçãs foram imersas em água fria $\left(3^{\circ} \mathrm{C}\right.$ por 2 minutos) para a remoção do calor, evitando o cozimento do produto.

Foram determinadas, em triplicata, a acidez, com os resultados expressos em \% de ácido málico e o pH inicial das amostras de acordo com a metodologia proposta pelo Instituto Adolfo 
Lutz (2008). As maçãs foram cortadas longitudinalmente com o auxílio de faca e expostas às condições ambientais. A vitamina $\mathrm{C}$ presente nas amostras foi quantificada a cada 15 minutos, por 2 horas (tempos de 0 a 8). $\mathrm{O}$ teor de vitamina $\mathrm{C}$ foi determinado segundo o método apresentado pela AOAC (1997), em que 0,5 g das amostras foram homogeneizadas em $50 \mathrm{~mL}$ de ácido oxálico por 3 minutos. A solução resultante foi titulada com DCFI (50 mg de DCFI e $20 \mathrm{mg}$ de bicarbonato de sódio em $100 \mathrm{~mL}$ de água destilada). O indicador DCFI foi padronizado com $10 \mathrm{~mL}$ de uma solução padrão de $10 \mathrm{mg} .100 \mathrm{~mL}^{-1}$ de ácido L-ascórbico. O ponto final da titulação foi definido no momento em que a solução titulada apresentou coloração rosa, reservando-se um período de 15 segundos para a confirmação do ponto de viragem.

Para o cálculo da quantidade de vitamina $\mathrm{C}$ presente nas amostras, foi aplicada a Equação 1:

$$
C=\frac{V \times f \times 100}{m}
$$

Sendo f calculado pela Equação 2:

$$
f=\frac{10 x c}{p}
$$

Em que "C" é a quantidade de vitamina (mg) presente em 100 g de amostra, "p" é o volume (mL) gasto de indicador - DCFI que reage com $10 \mathrm{~mL}$ de uma solução padrão de AA cuja concentração é "c" (mg.mL $\left.{ }^{-1}\right)$. "V" é o volume $(\mathrm{mL})$ de indicador - DCFI utilizado na titulação do extrato de amostra e "m" é a quantidade de amostra (g) utilizada na extração.

Para a análise dos fatores qualitativos foi realizado o esquema fatorial ( 8 × 3 ) utilizandose o programa computacional ASSISTAT versão 7.7 beta (SILVA e AZEVEDO, 2016). A comparação das médias foi feita pelo teste de Tukey a de 5\% de probabilidade de erro. Para a análise dos fatores quantitativos, foi realizada a regressão polinomial de segunda ordem e o ajuste do modelo aos dados experimentais expresso pelo coeficiente de determinação $\left(\mathrm{R}^{2}\right)$ em que houve efeito significativo do tempo.

\section{Resultados e Discussões}

Os médias para acidez total titulável e pH estão apresentadas na Tabela 1. Observa-se que, as amostras não apresentaram diferenças significativas entre si. Os valores de ATT e pH 
corroboram com Alves et al. (2017) encontrando valores de 0,20\% e 5,04 para maçãs variedade fuji, e próximos encontrados por Moura et al., (2012), ao caracterizarem maçãs in natura proveniente do Estado do Ceará, onde encontraram valores de 0,275 e 3,66 para estes mesmos parâmetros, respectivamente.

Tabela 1 - Médias e desvios padrão da ATT e pH das maçãs

\begin{tabular}{cccc}
\hline & Sem branqueamento & $70^{\circ} \mathrm{C}$ por 5 minutos & $70^{\circ} \mathrm{C}$ por 10 minutos \\
\hline ATT $\%$ ác. málico $)$ & $0,139 \pm 0,07 \mathrm{a}$ & $0,201 \pm 0,06 \mathrm{a}$ & $0,193 \pm 0,00 \mathrm{a}$ \\
$\mathrm{pH}$ & $5,04 \pm 0,03 \mathrm{a}$ & $5,02 \pm 0,12 \mathrm{a}$ & $5,01 \pm 0,10 \mathrm{a}$
\end{tabular}

*Médias com letras iguais na mesma linha não diferem estatisticamente entre si de acordo com o teste de Tukey a $5 \%$ de probabilidade. Fonte própria (2019).

Na Tabela 2 estão presentes os valores de vitamina C nas maçãs fuji. De acordo com a temperatura (médias apresentadas nas colunas), houve variações significativas em todos os tratamentos testados, a maçã sem branqueamento apresentou os maiores teores de vitamina C, seguida pelo tratamento a $70^{\circ} \mathrm{C}$ por 5 minutos e pelo tratamento a $70^{\circ} \mathrm{C}$ por 10 minutos. Sabese que diversos trabalhos vêm verificando que o efeito do binômio tempo x temperatura sobre a degradação de ácido ascórbico, como observado por Lima et al. (2010), em polpa de caju e por Mercali et al. (2012, 2014), em polpa de acerola As diferenças na concentração desta vitamina em ambos os tratamentos, significa que a estabilidade do ácido ascórbico é afetada pelo branqueamento utilizado e pelo tempo de exposição das maçãs.

De acordo com os dados da tabela de composição de alimentos norte-americana (USDA, 2011), para a maçã crua e com casca, o teor de vitamina $C$ calculado é de $460 \mathrm{~g} \cdot 100^{-1}$, tal valor apresenta-se bem acima dos encontrados nesse estudo. Essa diferença pode ser creditada a fatores genéticos (diferenças de variedade) e ao manejo pré e pós-colheita (estádio de maturação, condições de estocagem) (SPINOLA et al., 2013).

Tabela 2 - Médias e desvios padrão dos teores de vitamina C (g.100-1) em maçãs variedade fuji

\begin{tabular}{cccc}
\hline Tempo & Sem branqueamento & $70^{\circ} \mathrm{C}$ por 5 minutos & $70^{\circ} \mathrm{C}$ por 10 minutos \\
\hline 0 & $109,320 \pm 0,89 \mathrm{aA}$ & $102,500 \pm 0,29 \mathrm{aB}$ & $81,450 \pm 0,90 \mathrm{aC}$
\end{tabular}




$\begin{array}{lccc}1 & 108,021 \pm 1,25 \mathrm{aA} & 102,336 \pm 0,89 \mathrm{aB} & 80,096 \pm 0,70 \mathrm{aC} \\ 2 & 106,452 \pm 0,65 \mathrm{aA} & 100,835 \pm 1,40 \mathrm{aB} & 79,460 \pm 0,70 \mathrm{aC} \\ 3 & 101,374 \pm 1,05 \mathrm{bA} & 92,287 \pm 1,44 \mathrm{bB} & 72,919 \pm 0,89 \mathrm{bC} \\ 4 & 101,215 \pm 1,40 \mathrm{bA} & 92,356 \pm 1,75 \mathrm{bB} & 72,165 \pm 1,62 \mathrm{bC} \\ 5 & 90,230 \pm 1,28 \mathrm{cA} & 80,090 \pm 1,08 \mathrm{cB} & 66,930 \pm 0,32 \mathrm{cC} \\ 6 & 84,786 \pm 1,67 \mathrm{dA} & 72,189 \pm 1,39 \mathrm{~dB} & 64,068 \pm 0,78 \mathrm{cdC} \\ 7 & 79,817 \pm 1,30 \mathrm{eA} & 69,746 \pm 0,46 \mathrm{~dB} & 62,710 \pm 1,22 \mathrm{dC} \\ 8 & 77,236 \pm 0,72 \mathrm{eA} & 70,147 \pm 0,35 \mathrm{~dB} & 61,907 \pm 1,43 \mathrm{dC}\end{array}$

* Médias com letras iguais nas mesmas linhas e nas colunas não diferem estatisticamente entre si de acordo com 0 teste de Tukey a 5\% de probabilidade. Fonte própria (2019).

Na Figura 1 tem-se o gráfico da regressão polinomial de segunda ordem do teor de vitamina $\mathrm{C}$ em função do tempo para a mação sem branqueamento. Ocorreu redução de 32,08\% ao final das duas horas de exposição e a equação utilizada apresentou um modelo ajustável, podendo ser utilizada podendo representar de forma confiável os dados experimentais.

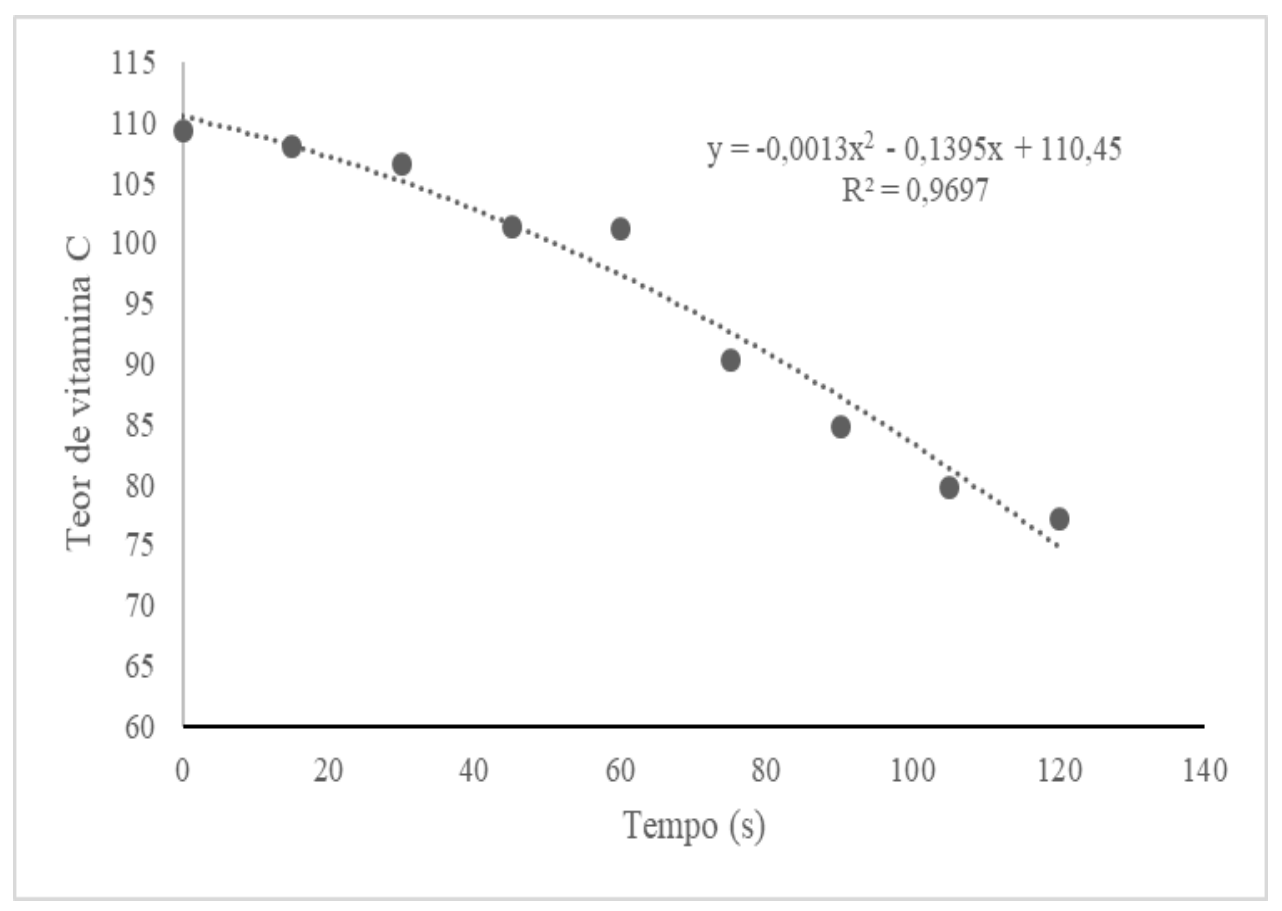

Figura 1 - Teor de vitamina $\mathrm{C}$ na maçã fuji sem branqueamento

Na Figura 2 tem-se o gráfico da regressão polinomial de segunda ordem do teor de vitamina $\mathrm{C}$ em função do tempo para a mação branqueada a $70^{\circ} \mathrm{C}$ por 5 minutos. Ocorreu 
redução de 32,35\% ao final das duas horas de exposição e a equação utilizada apresentou um modelo ajustável, podendo ser utilizada podendo representar de forma confiável os dados experimentais.

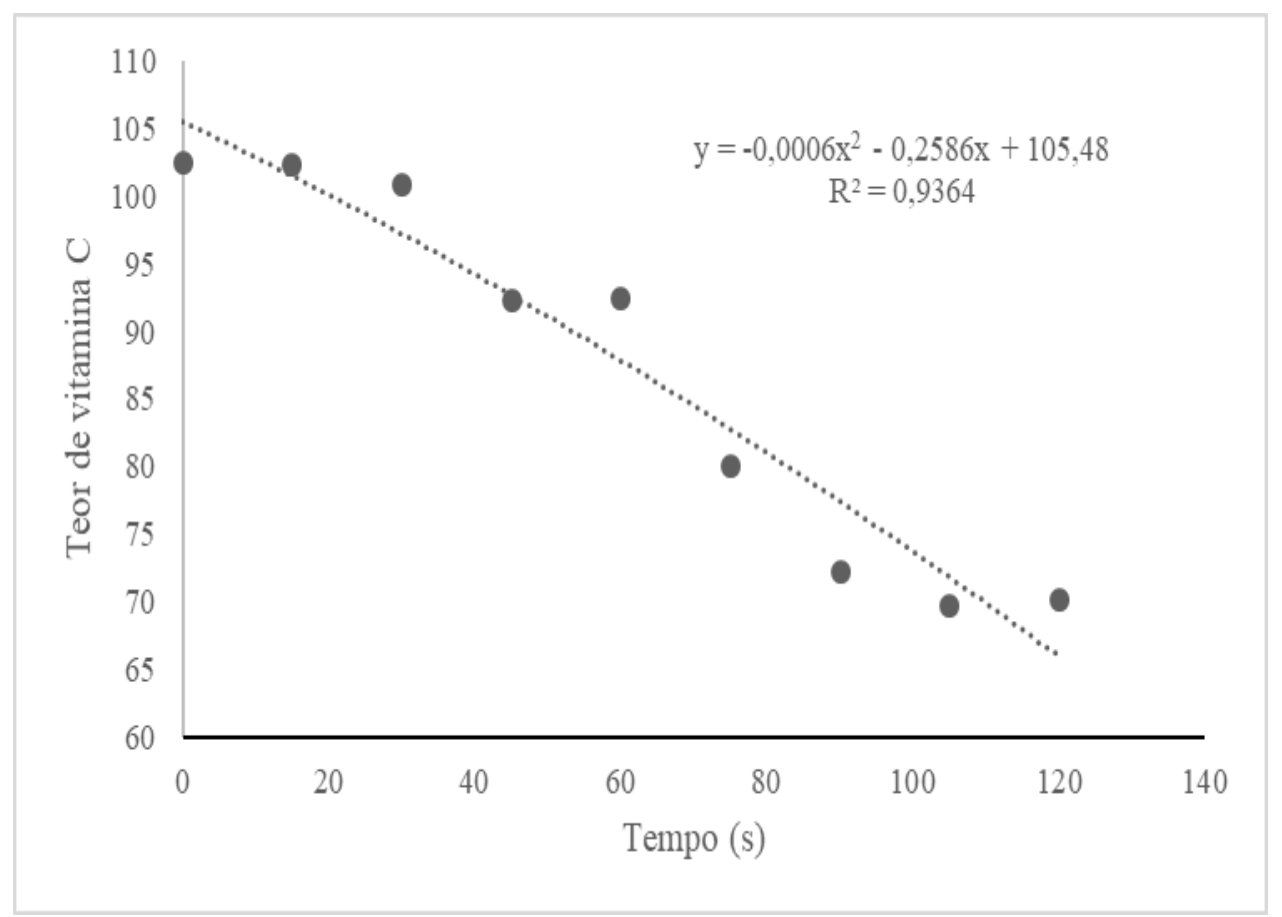

Figura 2 - Teor de vitamina $\mathrm{C}$ na maçã fuji branqueada a $70^{\circ} \mathrm{C}$ por 5 minutos

Na Figura 3 tem-se o gráfico da regressão polinomial de segunda ordem do teor de vitamina $\mathrm{C}$ em função do tempo para a mação branqueada a $70^{\circ} \mathrm{C}$ por 10 minutos. Ocorreu redução de $19,54 \%$ ao final das duas horas de exposição e a equação utilizada apresentou um modelo ajustável, podendo ser utilizada podendo representar de forma confiável os dados experimentais. 


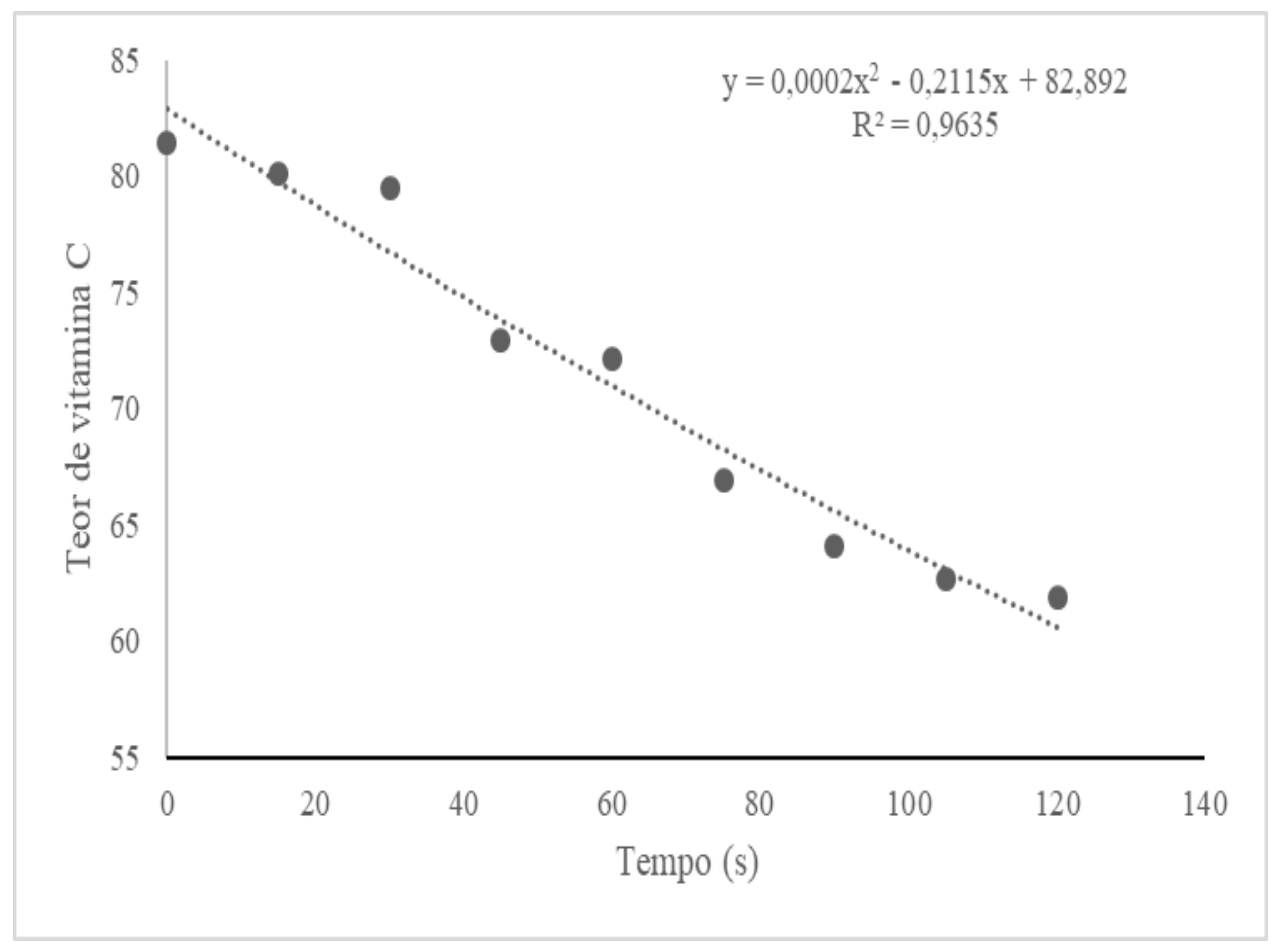

Figura 3 - Teor de vitamina $\mathrm{C}$ na maçã fuji branqueada a $70^{\circ} \mathrm{C}$ por 10 minutos

Observa-se que, as perdas de vitamina $\mathrm{C}$ nas maçãs foram significativas. De acordo com Lima et al. (2000b), a perda de vitamina C em frutas cortadas é causada principalmente pela incorporação de ar durante as etapas do processamento que favorece as reações aeróbicas de degradação, além da temperatura e tempo de armazenamento, sendo o uso de baixas temperaturas, condição imperativa para a retenção de vitamina $\mathrm{C}$ durante a estocagem. A degradação provavelmente deve-se às reações químicas envolvendo oxidação e formação de ácido desidroascórbico (DHAA), hidrólise do DHAA em ácido 2,3-dicicogulônico e a geração, por polimerização, de produtos inativos nutricionalmente (DEWANTO et al., 2002). Tais resultados podem ser comparados com o estudo realizado por Arroqui et al. (2002) avaliando as perdas de ácido ascórbico por difusão, durante o branqueamento de batata em água reciclada e destilada, em quatro diferentes tempos $(10,30,45$ e $60 \mathrm{~min})$ e três temperaturas $(65,80,93$ $\left.{ }^{\circ} \mathrm{C}\right)$. Os resultados mostraram que o aumento do tempo e da temperatura provocou perdas da vitamina, em ambas as condições analisadas.

Danieli et al. (2009) avaliaram a estabilidade da vitamina C em sucos de laranja industrializados e naturais. Os sucos naturais foram estáveis por até $14 \mathrm{~h}$ de armazenamento refrigerado, enquanto os industrializados não apresentaram perda significativa de ácido ascórbico por até $72 \mathrm{~h}$. 
Cunha et al. (2014), ao avaliarem a estabilidade do ácido ascórbico em sucos de frutas comerciais recém-preparados de laranja, abacaxi com hortelã e melancia, armazenados sob refrigeração e em temperatura ambiente, perceberam que não houve diferença significativa na estabilidade da vitamina em sucos armazenados em temperaturas entre 6 e $30^{\circ} \mathrm{C}$, no período testado. Com relação ao tempo, o ácido ascórbico foi estável por 24h no suco de laranja, já no suco de abacaxi com hortelã e no de melancia, houve decréscimo significativo após $8 \mathrm{~h}$ de armazenamento.

\section{Conclusões}

A acidez e o pH das maçãs estão de acordo com a literatura, entretanto, o teor de vitamina $\mathrm{C}$ quantificado encontra-se abaixo do esperado. As maçãs apresentam diferenças significativas entre os tratamentos sendo o maior teor observado na amostra sem branqueamento em relação as demais. De acordo com o tempo de branqueamento, as perdas quantificadas foram de 32,08, 32,35 e 19,54\% para as amostras não branqueadas, branqueadas a $70^{\circ} \mathrm{C}$ por 5 minutos e branqueadas a $70^{\circ} \mathrm{C}$ por 10 minutos, respectivamente, o que é uma desvantagem, visto que esta perda é considerada um dos parâmetros para avaliar a eficiência do pré-processamento.

\section{Referências}

ALVES, J.A.; NASSUR, R.C.M.R; PIRES, C.R.F; ALCÂNTARA, E.M.; GIANNONI, J.A.; LIMA, L.C.O. Cinética de degradação de vitamina C em mangas "Palmer" minimamente processadas armazenadas em diferentes temperaturas. Ciência e Agrotecnologia, v. 34, n. 3, p. 714-721, 2010.

ALVES, K. M. A.; LIMA, H. S. C.; MORAES, M. S. A.; PEREIRA, M. S.; CAVALCANTI, M. S. Comparação das qualidades físico-quimicas da maçã Argentina red com a maçã nacional fuji. In: II Congresso Brasileiro de Ciências da Saúde. Campina Grande - PB, 2234-238, 2017.

AOAC. ASSOCIATION OF OFFICIAL ANALYTICAL CHEMISTS. Official Methods of Analysis of AOAC International. 16a . ed. Maryland: AOAC, 1997. 1141p.

AROQUI, C. et al. Losses by diffusion of ascorbic acid during recycled water blanching of potato tissue. J. Food Eng., v.52, p.25-30, 2002.

CUNHA, K. D.; SILVA, P. R.; COSTA, A. L. F. S. F.; TEODORO, A. J.; KOBLITZ, M. G. B. Estabilidade de ácido ascórbico em sucos de frutas frescos sob diferentes formas de armazenamento. Braz. J. Food Technol, Campinas, v. 17, n. 2, p. 139-145, abr./jun. 2014 
DANIEli, F.; COSTA, L. R. L. G.; SILVA, L. C.; HARA, A. S. S.; SILVA, A. A. Determinação de Ácido Ascórbico em Amostras de Suco de Laranja in natura e Amostras Comerciais de Suco de Laranja Pasteurizado e Envasado em Embalagem Tetra Pak. Revista do Instituto de Ciências da Saúde, São Paulo, v. 27, n. 4, p. 361-365, 2009.

DEWANTO, V.; WU, X.; LIU, R.H. Processed sweet corn has higher antioxidant activity. Journal of Agricultural and Food Chemistry, v. 50, n. 17, p. 4959-4964, 2002

FERNÁNDEZ, A.; DOS SANTOS, M.; DA SILVA, D.; DE SOUSA, P.; MAIA, G. \& DE FIGUEIREDO, R. Chemical and physicochemical characteristics changes during passion fruit juice processing. Ciência e Tecnologia de Alimentos, v.31, n.3, p.747-751, 2011.

GEORGÉ, S.; TOURNIAIRE, F.; GAUTIER, H.; GOUPY, P.; ROCK, E. \& CARIS, C. Changes in the contents of carotenoids, phenolic compounds and vitamin $\mathrm{C}$ during technical processing and lyophilisation of red and yellow tomatoes. Food Chemistry, v.124, n.4, p.16031611, 2011.

INSTITUTO ADOLFO LUTZ. Métodos físico-químicos para análise de alimentos. Edição

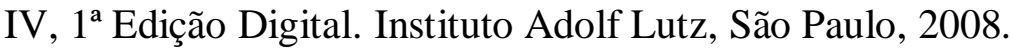

KALIORA, A. C.; DEDOUSSI, G. V. Z.; SCHMIDT, H. Dietary antioxidants in preventing atherogenesis, Atherosclerosis, v. 187, p. 1 - 17, 2006.

LAVRADA, L. Determinação da cinética de degradação térmica da vitamina c em polpa de acerola via aquecimento ôhmico. 2011, 44f. Trabalho de Conclusão de Curso (Engenharia Química). Universidade Federal do Rio Grande do Sul - UFRS (Porto Alegre). 2011.

LIMA, J. R.; ELIZONDO, N. J.; BOHUON, P. Kinetics of Ascorbic Acid Degradation and Colour Change in Ground Cashew Apples Treated at High Temperatures $\left(100-180^{\circ} \mathrm{C}\right)$. International Journal of Food Science and Technology, Oxford, v. 45, p. 1724-1731, 2010.

LIMA V.L.A. G; MELO, E.A; LIMA, L.S. Avaliação da qualidade de suco de laranja industrializado. Boletim CEPPA, v.18, n.1, p.95-104, 2000

MAIA, C. A.; SOUSA, P. H. M,; SANTOS, G. M.; SILVA, D. S.; FERNANDES, A. G.; PRADO, G. M. Efeito do processamento sobre componentes do suco de acerola. Ciência e Tecnologia Alimentos, Campinas, 27(1): 130-134, 2007.

MELLO, L. M. R.; JÚNIOR, L. B. Mercado nacional e internacional. In: GIRARDI, C. L. (ed.). Maçã: Pós-colheita. Bento Gonçalves: Embrapa Uva e Vinho, p. 10-23, 2004. (Série Frutas do Brasil, 39).

MERCALI, G. D.; JAESCHKE, D. P.; TESSARO, I. C.; MARCZAK, L. D. F. Study of Vitamin C Degradation in Acerola Pulp During Ohmic and Conventional Heat Treatment. LWT - Food Science and Technology, London, v. 47, n. 1, 91-95, 2012.

MERCALI, G. D.; SCHWARTZ, S.; MARCZAKA, L. D. F.; TESSAROA, I. C.; SASTRYC, S. Ascorbic Acid Degradation and Color Changes in Acerola Pulp During Ohmic Heating: 
Effect of Electric Field Frequency. Journal of Food Engineering, New York, v. 123, p. 1-7. 2014.

MOURA, F. P. A.; NAZÁRIO, A. S. N.; LIMA, M. A.; MOREIRA, L. F.; HOLANDA, N. V.; SOUZA, P. A. Caracterização físico-química de maçãs desidratadas. In: Congresso Norte Nordeste de pesquisa e inovação. 7. 2012. Palmas - Tocantins. Anais... Palmas. VII CONNEPI. 2012.

NAGAOKA, $\mathrm{S}$. et al. Kinetics of the reaction by vitamin $\mathrm{E}$ is regenerated which natural by vitamin C, Chem. Phys. Lipids, v. 146, p. 26 - 32, 2007.

ORSO, E. Estudo dos fatores que influenciam a eficiência do Branqueamento em couveflor. Bento Gonçalves, 2011, 33f. Trabalho de Conclusão de Curso (TCC), Instituto Federal de Educação, Ciência e Tecnologia, Rio Grande do Sul -IFRS.

REBOUCHE, C. J. Ascorbic acid and carnitine biosynthesis, American Journal of Clinical Nutrition, v. 54, p. 1147S - 1147S, 1991.

SILVA, F. DE A. S. E.; AZEVEDO, C. A. V. Principal Components Analysis in the Software Assistat-Statistical Attendance. In: WORLD CONGRESS ON COMPUTERS IN AGRICULTURE, 7, Reno-NV-USA: American Society of Agricultural and Biological Engineers, 2009.

SPINOLA, V.; BERTA, B.; CÂMARA, J. S.; CASTILHO, P. C. Effect of Time and Temperature on Vitamin C Stability in Horticultural Extracts. UHPLC-PDA vs. Iodometric Titration as Analytical Methods. LWT - Food Science and Technology, London, v. 50, n. 2, p. 489-495, 2013.

SUCUPIRA, Natália Rocha; XEREZ, Ana Caroline Pinheiro; DE SOUSA, Henrique Machado. Perdas vitamínicas durante o tratamento térmico de alimentos. UNOPAR Científica Ciências Biológicas e da Saúde, v. 14, n. 2, 2014.

TARRAGO-TRANI, M. T.; PHILlIPS, K. M.; COTTY, M. Matrix-Specifi c Method Validation for Quantitative Analysis of Vitamin C in Diverse Foods. Journal of Food Composition and Analysis, London, v. 26, n. 1-2, p. 12-25, 2012.

TAVARES, J.T.Q; SILVA,C.L; CARVALHO,L.A.; SILVA, M.A.; SANTOS, C.M.G. Estabilidade do Ácido ascórbico em suco de laranja submetido a diferentes tratamentos. Magistra, Cruz das Almas-BA, v. 12, n. 1/2, 2000.

TEIXEIRA, M.; MONTEIRO, M. Caracterização físico-química e sensorial de suco de laranja processado. In: CONGRESSO BRASILEIRO DE CIÊNCIA E TECNOLOGIA DE ALIMENTOS, 19, 2004, Recife. Anais... Recife: SBCTA, 2004.

UMME, A. et al. Characteristics of soursop natural puree and determination of optimum conditions for pasteurization. Food Chemistry, v. 58, p. 119-124, 1997. 
UNITED STATES DEPARTMENT OF AGRICULTURE - USDA.mNational Nutrient Data Base for Standard Reference. Washington: USDA, 2011. Disponível em: $<$ http://ndb.nal.usda.gov/>.

VASCONCELOS, M. A. S.; FILHO, A. B. M. Conservação de alimentos. Disponível em: http://redeetec.mec.gov.br/images/stories/pdf/eixo_prod_alim/tec_alim/181012_con_alim.pdf >. Acesso em: 07 Jul 2019.

YAMASHITA, F; BENASSI, M.T; TONZAR, A.C; MORIYA, S; FERNANDES, J.G. Produtos de acerola: estudo da estabilidade de vitamina C. Ciênc. Tecnol. Alimentos, v. 23, n.1, p.92-94, 2003.

ZHANG, D; HAMAUZU, Y. Phenolics, ascorbic acid, carotenoids and antioxidant activity of broccoli and their changes during conventional and microwave cooking. Food Chemistry, London, v. 88, n. 4, p. 503-509, 2004. 